\title{
ÁBALOS + SENTKIEWICZ
}

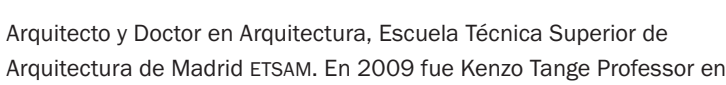
Harvard University Graduate cshool of Design, donde es proféfsoror invitad

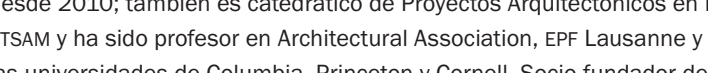
Abalos \& Herereros (1984-2006) y de ébalos + Sentkiewicz arquitectos

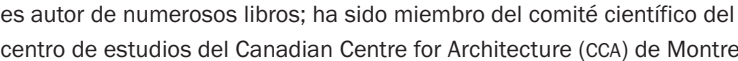
y del consejo de dirección de Barcelona Institute of Architecture. Desde 2002 es director del Laboratatri de Técnicicas y Paisjajes Contemporáneos

\section{Renata Sentkiewicz I P
Arquitectura de Madrid.}

Wuitecta, Politechnika Krakowska. En 2011 fue Adjunct Professor en Arquitectónicos en la Escuela Técrica Superior de Arautitectura de Madrid ETSAM y desde 2002 del Laboratorio de Técnicas Y Paisajes

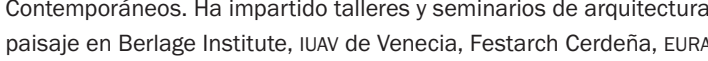
conc, Universididad Nacional de Colombia y cEu, entre otras instituciones En 1999 comennzo a colaborar en Abalos \& Herreros, donde fue asociac partir de 2001 . Es socia fundador
miembro de Zero Energy Alliance.

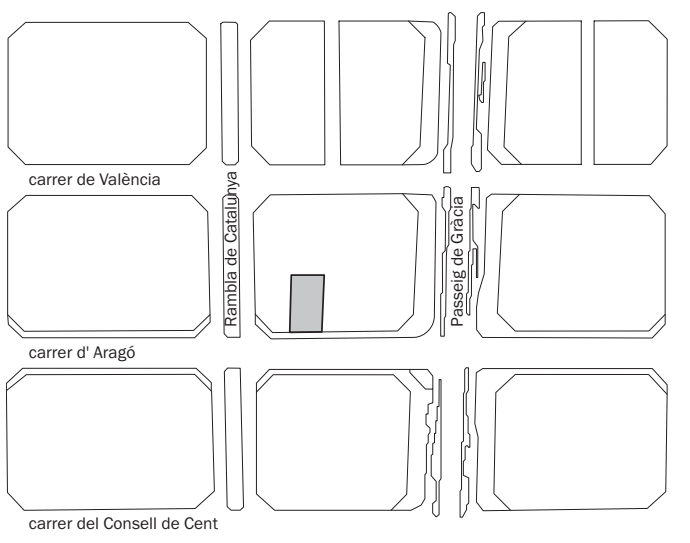

$\bigcup_{\substack{\text { E. 1: } 7.500 \\ \text { Planta emplazamiento }}}$

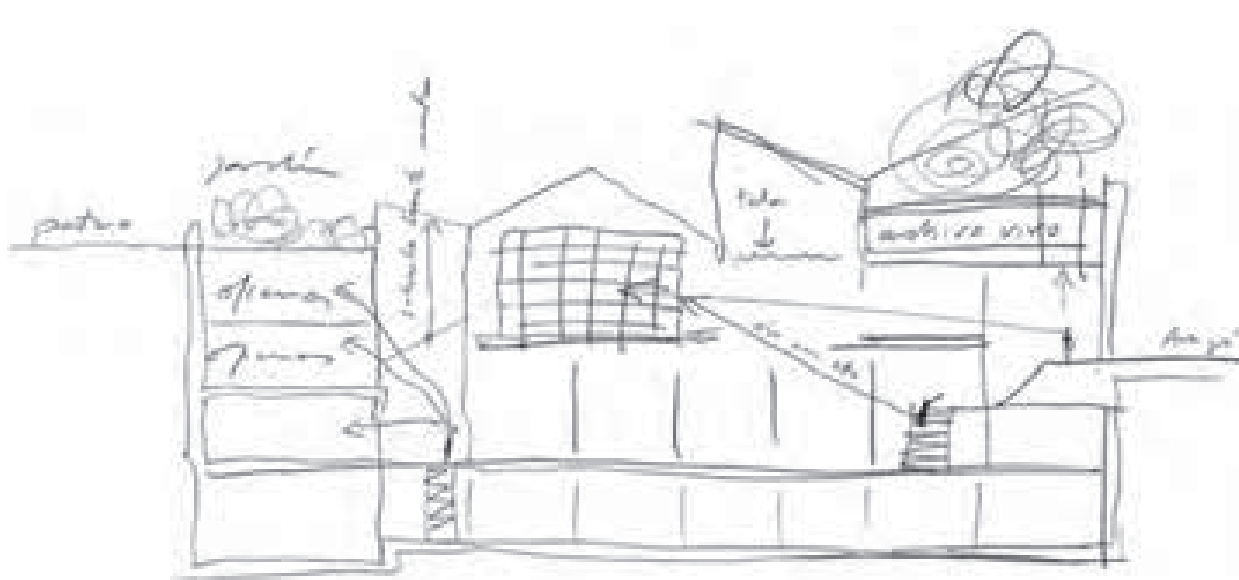

k tode mino de una institución cultural da pie para la colonización del corazón barceloness, para abrit una realidad urbana - España, centro cuttural termodinámica, ensanche The extension of a cultural
institution established in the Barcelona Ensanche causes the
occupation of the inner rareas Of the block and brings out the
intimacy of that urban realm. Keywords: Arcritibecture - - Spain.
cultural center, thermodynamis Barcelona ensanche.

FUNDACIÓ ANTONI TÀPIES BARCELONA, 2005-2009

Mrimer objeivo dia de seguridad y evacuación en una mejora integral del conjunto. Se pretende abrir todo el edificio historico al público, con nuevas áreas de exposición, de archivo y educativas, y concentrar las áreas administrativas en un pabellon de tres plantas ubicado en el fondo de $\mathrm{l}$ Pero la nueva Fundació Antoni Tàpies aspan

contribuir con la consolidación de una nueva seos, entendidos como centros de producción cultural. Se plantea una espacialidad multiple, adaptada a la diversidad de las prácticas valor del patrimonio heredado y a la disolución atonosférica. Esto en fuor de la intensificción de la experiencia del visitante, a quien se ofrece visualizar todo el complejo entramado programático que soporta la producción cultural y el propio edificio modernista como parte misma del sistema expositivo.

De

vención de Roser Amadó y Lluís Domènech de 1987-1990, en especial la instalación de la obra de Antoni Tàpies en la cubierta -Núvol icadira (Nubey silla, 1990)-, se incorpora ahora otra pieza de Tàpies a nuevos diálogos con el patio de manzana del plan Cerdà En términos medioambientales, la ampliación supone una ra sustancial al uso de la iluminación natural del edificio original. También al factor de forma del conjunto, al englobar la intervención en un volumen único en beneficio del control y reducción de los

Corte AA
$E .1: 500$ 


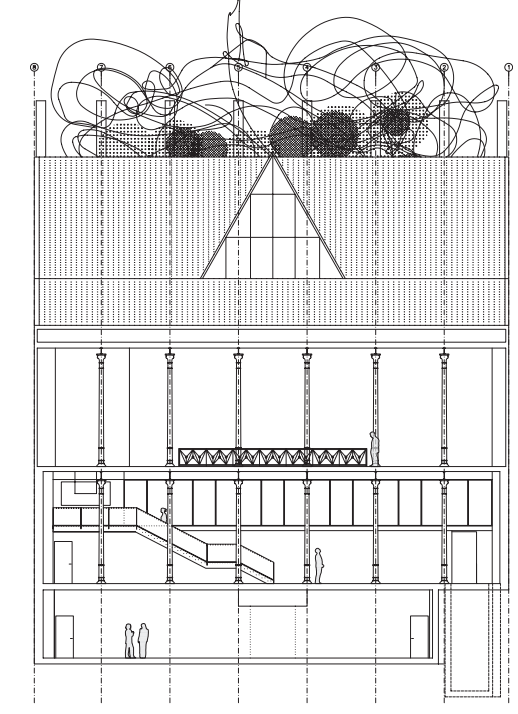

Core 88
E.1.500
$\varepsilon$

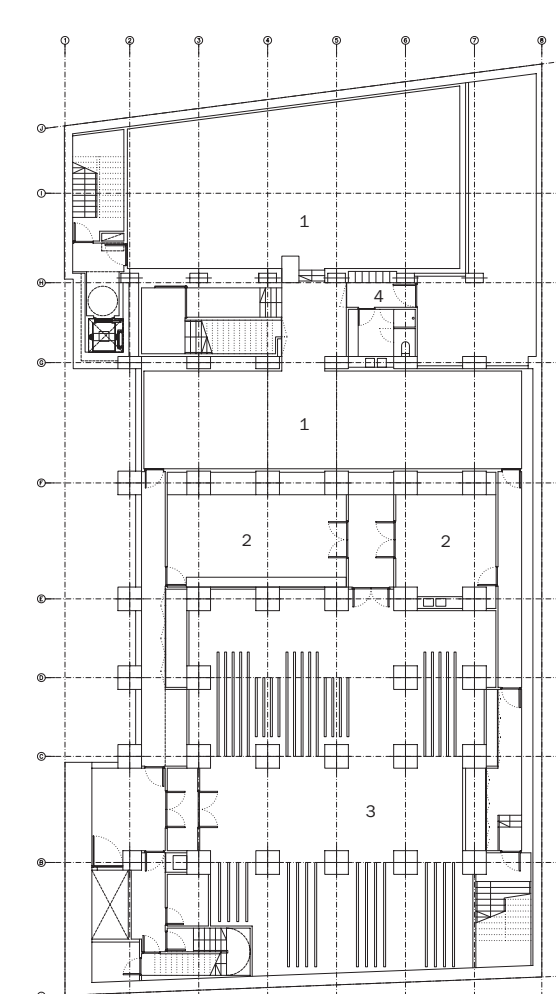

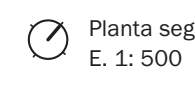

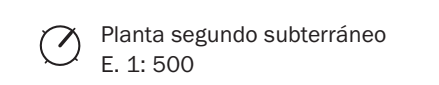

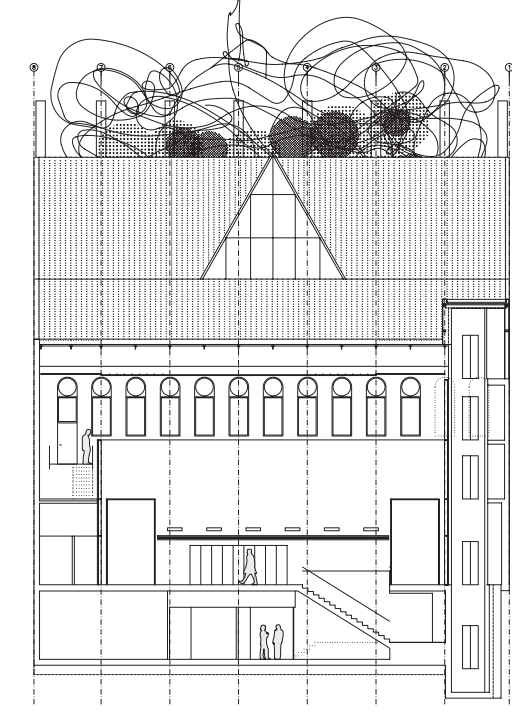

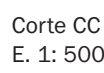

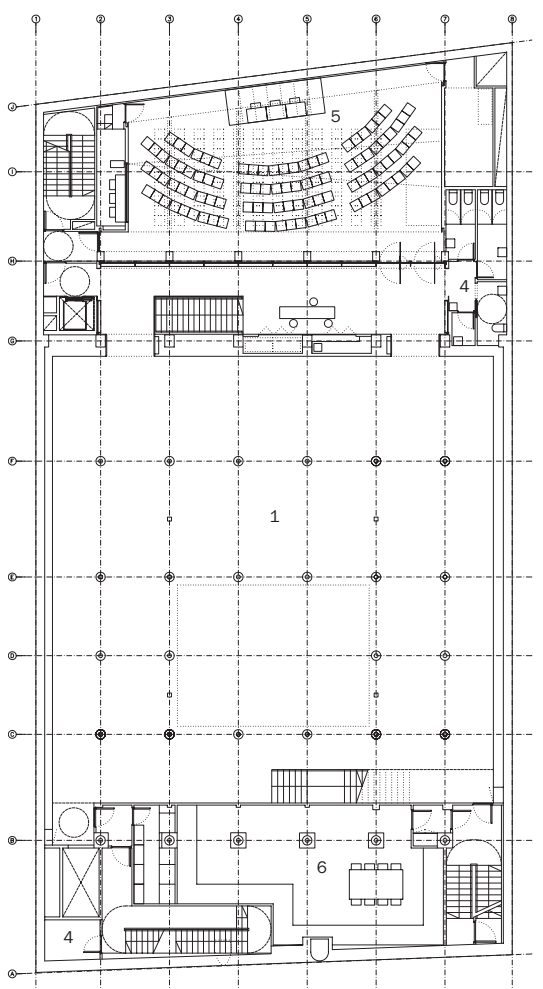

Planta primer subterráneo
E.1: 500

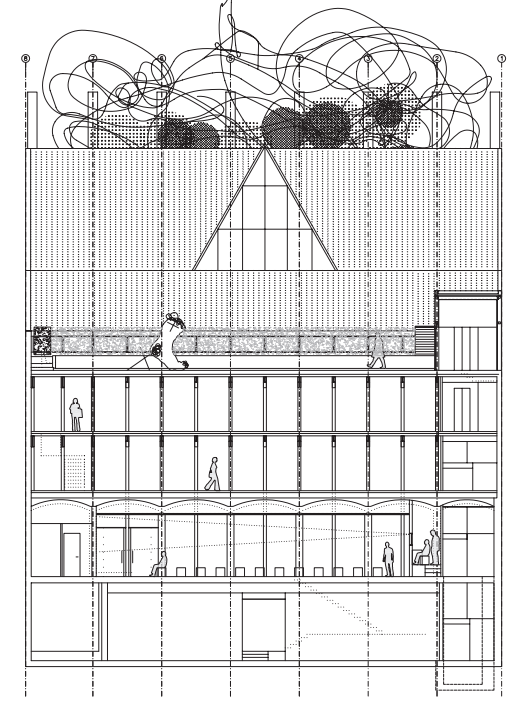

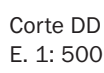

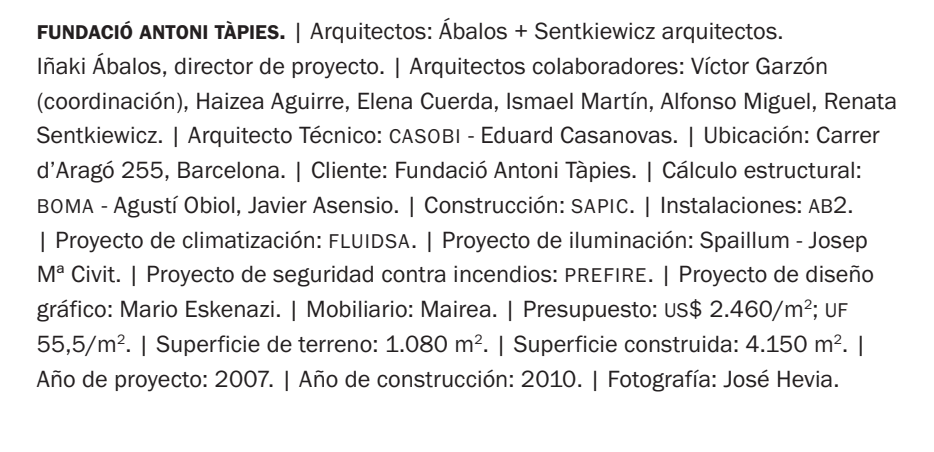

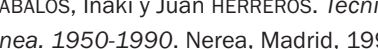

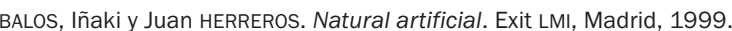

Les, Irakki. La buena vida. Editorial Gustavo Gill, Barcelona, 2000.

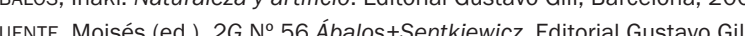

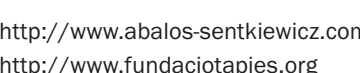

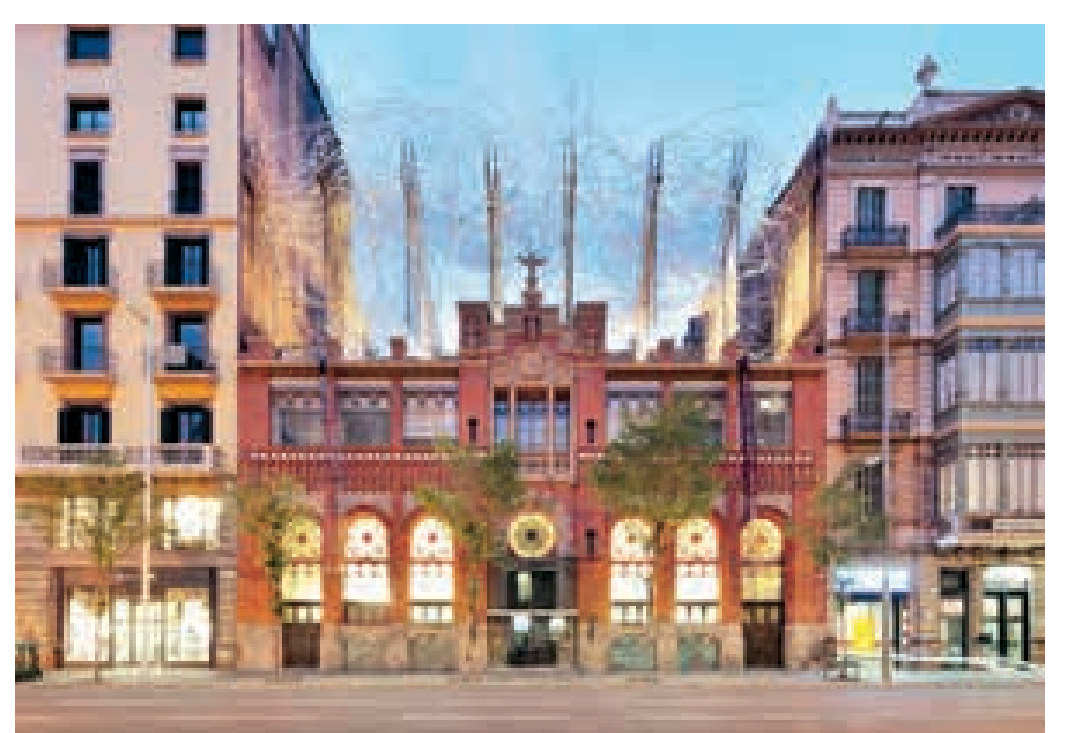

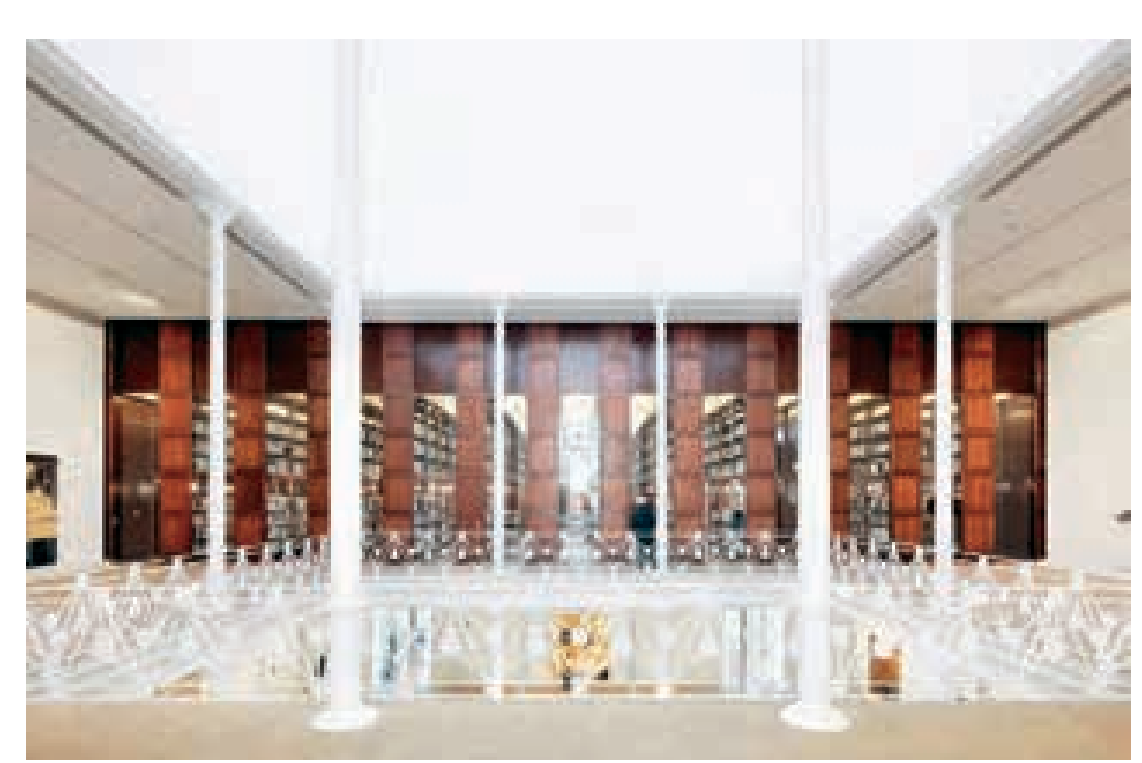

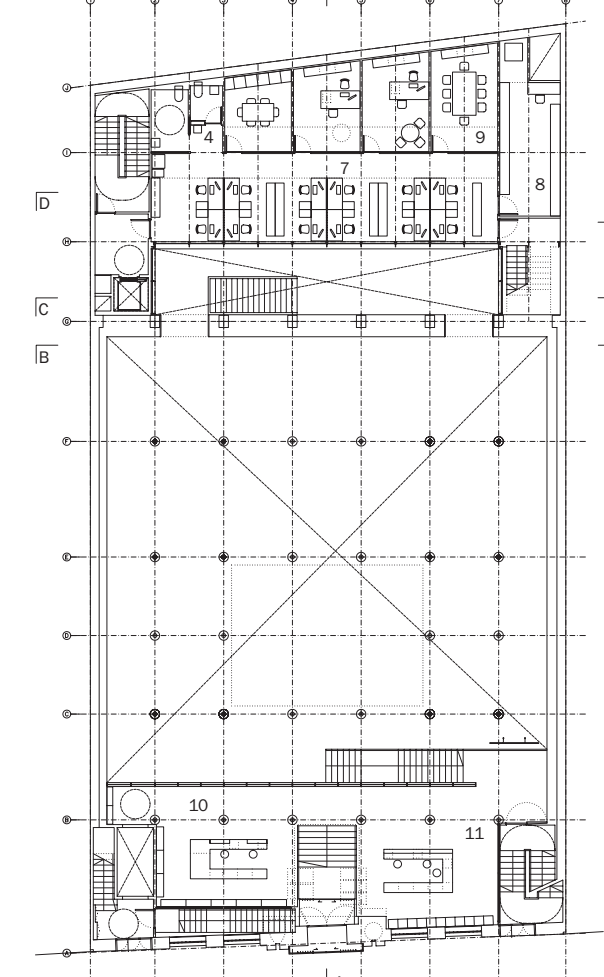

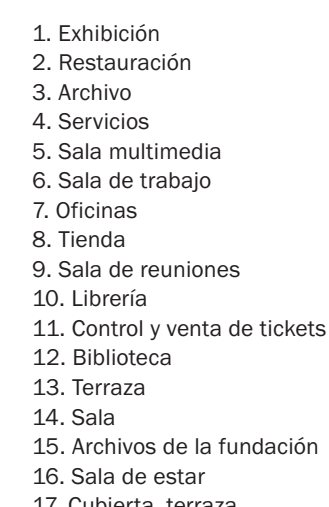

Panara nivel acceses

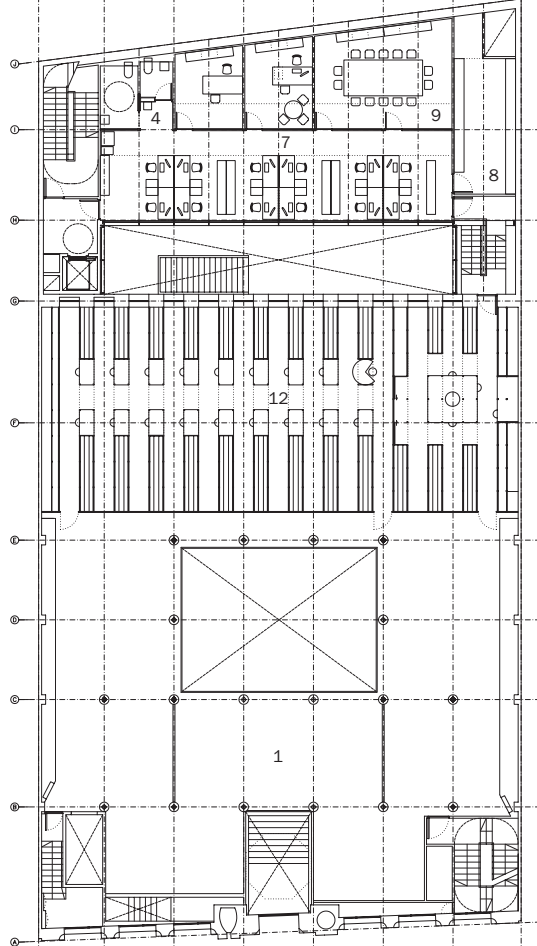

Planta primer nivel

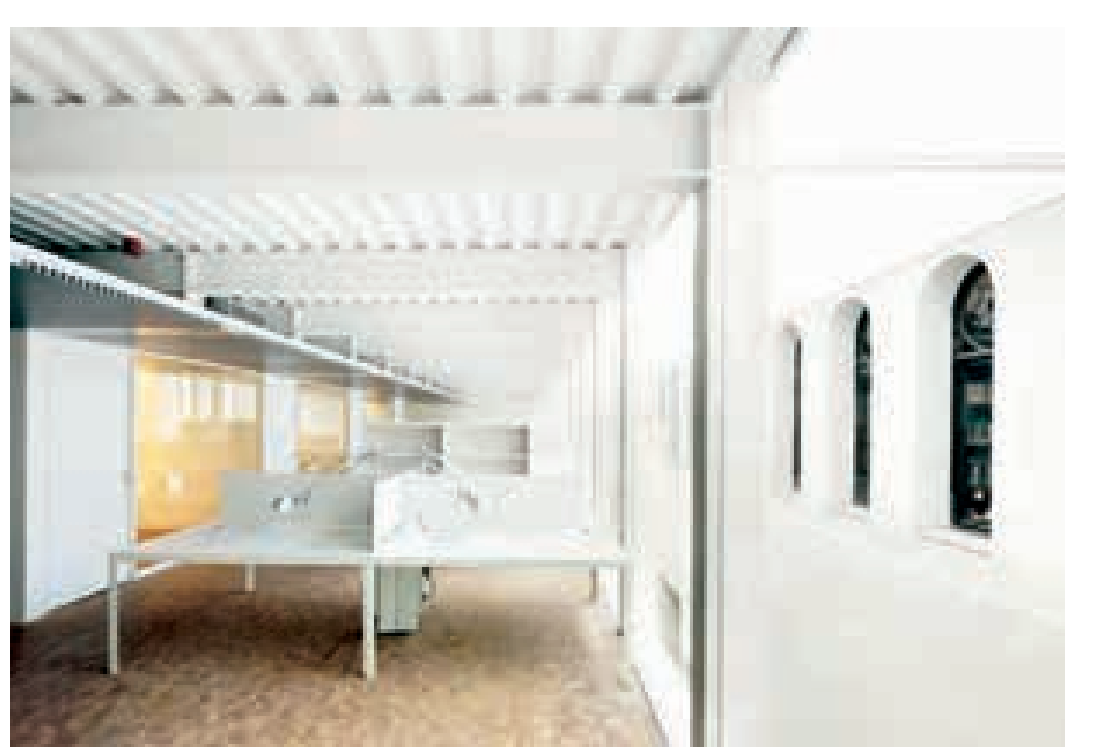

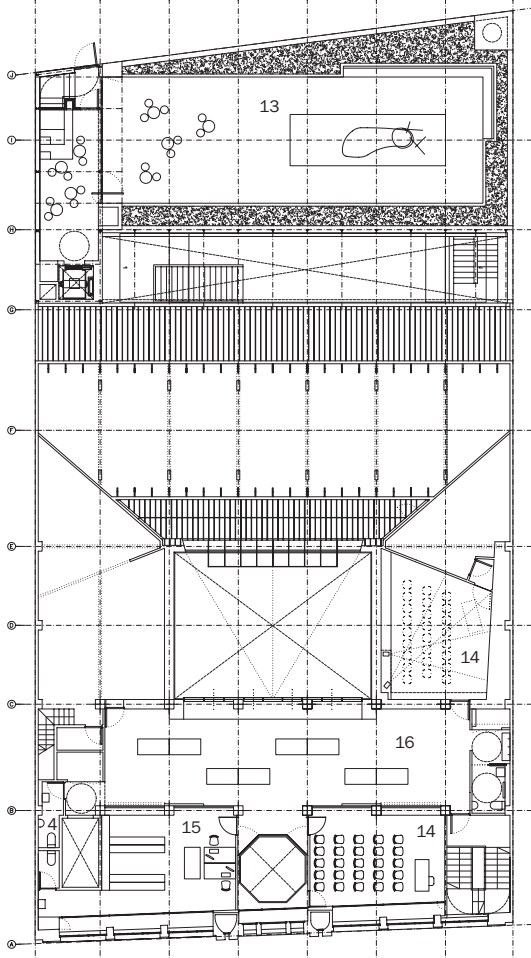

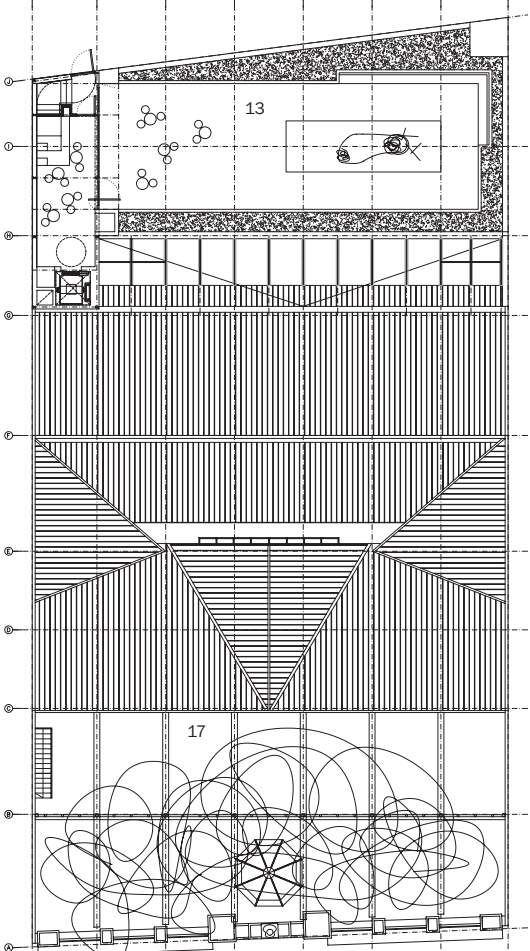

Planta segundo nivel
E.1. 1 . 00
Planta cubierta
E.1:500

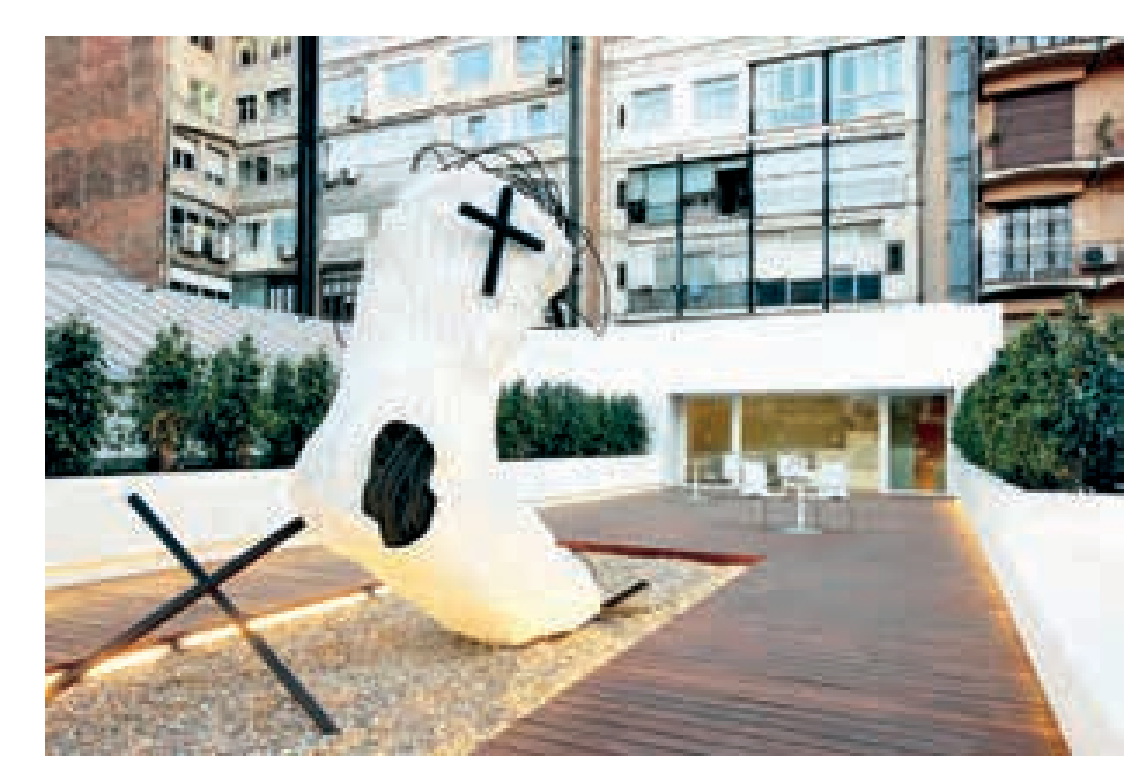

